TR ANSACTIONS OF THE

AMERICAN MATHEMATICAL SOCIETY

Volume 181, July 1973

\title{
SUCCESSIVE REMAINDERS OF THE NEWTON SERIES
}

BY

\author{
G. W. CROFTS AND J. K. SHAW
}

ABSTRACT. If $f$ is analytic in the open unit disc $D$ and $\boldsymbol{\lambda}$ is a sequence of points in $D$ converging to 0 , then $f$ admits the Newton series expansion $f(z)=f\left(\lambda_{1}\right)+\Sigma_{n=1}^{\infty} \Delta_{\lambda}^{n} f\left(\lambda_{n+1}\right)\left(z-\lambda_{1}\right)\left(z-\lambda_{2}\right) \cdots\left(z-\lambda_{n}\right)$, where $\Delta_{\lambda}^{n} f(z)$ is the $n$th divided difference of $f$ with respect to the sequence $\lambda$. The Newton series reduces to the Maclaurin series in case $\lambda_{n} \equiv 0$. The present paper investigates relationships between the behavior of zeros of the normalized remainders $\Delta_{\lambda}^{k} f(z)=\Delta_{\lambda}^{k} f\left(\lambda_{k+1}\right)+\Sigma_{n=k+1}^{\infty} \Delta_{\lambda}^{n} f\left(\lambda_{n+1}\right)\left(z-\lambda_{k+1}\right) \cdots\left(z-\lambda_{n}\right)$ of the Newton series and zeros of the normalized remainders $\sum_{n=k}^{\infty} a_{n} z^{n-k}$ of the Maclaurin series for $f$. Let $C_{\lambda}$ be the supremum of numbers $c>0$ such that if $f$ is analytic in $D$ and each of $\Delta \underset{\lambda}{k} f(z), 0 \leq k<\infty$, has a zero in $|z| \leq c$, then $f \equiv 0$. The corresponding constant for the Maclaurin series $\left(C_{\lambda}\right.$, where $\left.\lambda_{n} \equiv 0\right)$ is called the Whittaker constant for remainders and is denoted by $W$. We prove that $C_{\lambda} \geq W$, for all $\lambda$, and, moreover, $C_{\lambda}=W$ if $\lambda \in l_{1}$. In obtaining this result, we prove that functions $f$ analytic in $D$ have expansions of the form $f(z)=\Sigma_{n=0}^{\infty} \Delta_{\lambda}^{n} f\left(z_{n}\right) C_{n}(z)$, where $\left|z_{n}\right| \leq W$, for all $n$, and $C_{n}(z)$ is a polynomial of degree $n$ determined by the conditions $\Delta_{\lambda}^{j} C_{k}\left(z_{j}\right)=\delta_{j k}$.

1. Introduction. Let $f$ be analytic in the open unit disc $D$ and let $\lambda$ denote a sequence of points in $D$. The Newton series for $f$ is given by

$$
f(z)=f\left(\lambda_{1}\right)+\sum_{n=1}^{\infty} \Delta_{\lambda}^{n} f\left(\lambda_{n+1}\right)\left(z-\lambda_{1}\right)\left(z-\lambda_{2}\right) \cdots\left(z-\lambda_{n}\right)
$$

where $\Delta_{\lambda}^{n} f$ denotes the $n$th divided difference of $f$ with respect to $\lambda$ :

$$
\Delta_{\lambda}^{0} f(z)=f(z), \quad \Delta_{\lambda}^{n} f(z)=\frac{\Delta_{\lambda}^{n-1} f(z)-\Delta_{\lambda}^{n-1} f\left(\lambda_{n}\right)}{z-\lambda_{n}}, \quad n=1,2,3, \cdots
$$

The series in (1.1) converges uniformly to $f$ on compact subsets of $D$ for each $\lambda \in c_{0}$, the space of complex sequences converging to $0([10],[4])$. Note that (1.1) reduces to the Maclaurin series for $f$ in case $\lambda_{n} \equiv 0$. If $\lambda$ is the constant sequence $\lambda_{n} \equiv z_{0}$, then (1.1) is simply the Taylor series for $f$, expanded about $z_{0}$. In this case, convergence is guaranteed only in $\left|z-z_{0}\right|<1-\left|z_{0}\right|$.

Presented to the Society, August 30, 1972; received by the editors September 5, 1972. AMS (MOS) subject classifications (1970). Primary 30A98, 30A40; Secondary 30A16.

Key words and phrases. Newton series, zeros of remainders, extremal functions, matrix transformations. 
In the present paper, we consider the behavior of zeros of the successive normalized remainders $\Delta_{\lambda}^{k} f\left(\lambda_{k+1}\right)+\sum_{n=k+1}^{\infty} \Delta_{\lambda}^{n} f\left(\lambda_{n+1}\right)\left(z-\lambda_{k+1}\right) \cdots\left(z-\lambda_{n}\right)$, $0 \leq k<\infty$, of the Newton series. In view of the identity

$$
\Delta_{\lambda}^{k} f(z)=\Delta_{\lambda}^{k} f\left(\lambda_{k+1}\right)+\sum_{n=k+1}^{\infty} \Delta_{\lambda}^{n} f\left(\lambda_{n+1}\right)\left(z-\lambda_{k+1}\right) \cdots\left(z-\lambda_{n}\right),
$$

which follows from a simple induction argument, our problem is equivalent to studying the zeros of the successive divided differences $\Delta_{\boldsymbol{\lambda}}^{k} f$ of $f$. For $\lambda \in c_{0}$, let $C_{\boldsymbol{\lambda}}$ denote the supremum of positive numbers $c$ such that if $f$ is analytic in $D$ and each of $\Delta_{\lambda}^{k} f(z), 0 \leq k<\infty$, has a zero in $|z| \leq c$, then $f \equiv 0$. The bound $C_{\lambda} \leq 1$ is part of the definition. In 1965, M. Pommiez [10] proved that $C_{\lambda} \geq .536$ for each $\lambda \in c_{0}$. Pommiez noted that $C_{\lambda}$ might be independent of $\lambda$ for suitably restricted sequences in $c_{0}$.

In the case when $\lambda$ is the null sequence $\nu\left(\nu_{n} \equiv 0\right), C_{\nu}$ has been determined ([1], [2], [3], [6]). Here, one considers the successive normalized remainders $\mathcal{S}^{k} f(z)=\sum_{n=k}^{\infty} f^{(n)}(0) z^{n-k} / n !(k=0,1,2, \ldots)$ of the Maclaurin series, or equivalently, the zeros of the shift operator $\mathfrak{S}^{k} f$. The constant $W(\mathfrak{S})$ is the supremum of positive numbers $c$ such that if $f$ is analytic in $D$ and each of $\mathcal{S}^{k} f(z), 0 \leq$ $k<\infty$, has a zero in $|z| \leq c$, then $f \equiv 0$. Clearly, we have

$$
C_{\nu}=W(\S)
$$

$W(\mathcal{S})$ is known as the Whittaker constant belonging to $\mathcal{S}$, and satisfies $.549<$ $W(\mathcal{S})<.562$. The following theorem, due to J. D. Buckholtz and J. L. Frank $([3],[2])$ completely characterizes $W(\mathfrak{S})$.

Theorem. Let $f(z)=\sum_{n=0}^{\infty} a_{n} z^{n}$ bave radius of convergence 1 and let $\epsilon>0$. Then

(i) infinitely many of the partial sums $\sum_{n=0}^{k} a_{n} z^{n}$ bave all their zeros in the $\operatorname{disc}|z| \leq W(\mathfrak{S})^{-1}+\epsilon$,

(ii) infinitely many of the remainders $\sum_{n=k}^{\infty} a_{n} z^{n-k}$ bave no zero in $|z| \leq$ $\left(W(\mathcal{S})^{-1}+\epsilon\right)^{-1}$,

(iii) $W(S)$ cannot be replaced by a larger number in either (i) or (ii).

Because of this result, the number $P=W(\delta)^{-1}$ is called the power series constant.

Following our remarks concerning the null sequence, it is natural to ask whether some extension of (1.4) holds for the nontrivial sequences in $c_{0}$. In this direction, our principal result is

Theorem 1. For each $\lambda \in c_{0}, C_{\boldsymbol{\lambda}} \geq W(\mathcal{S})$; moreover, $C_{\boldsymbol{\lambda}}=W(\mathfrak{S})$ for each $\lambda \epsilon$ $l_{1}=\left\{\mu: \Sigma\left|\mu_{n}\right|<\infty\right\}$. 
This is proved in $\$ 3$. To simplify notation in Theorem 1 and its proof, we will abbreviate $W(\mathcal{S})$ to $W$ and drop the subscript $\lambda$ from $\Delta_{\lambda}^{n}$ when no confusion is likely as to the particular sequence $\lambda$ under consideration.

A further characterization of $W$, and one which we will need, is obtained from the remainder polynomials $B_{n}\left(z ; z_{0}, z_{1}, z_{2}, \cdots, z_{n-1}\right)$. These are defined inductively by

$$
B_{0}(z)=1
$$

$$
B_{n}\left(z ; z_{0}, z_{1}, \cdots, z_{n-1}\right)=z^{n}-\sum_{k=0}^{n-1} z_{k}^{n-k} B_{k}\left(z ; z_{0}, z_{1}, \ldots, z_{k-1}\right) \text {, }
$$

for $n=1,2,3, \cdots$, where $\left\{z_{k}\right\}_{k=0}^{\infty}$ is a sequence of complex numbers. Let $H_{n}=$ $\max \left|B_{n}\left(0 ; w_{0}, w_{1}, \cdots, w_{n-1}\right)\right|$, where the maximum is taken over all sequences $\left\{w_{k}\right\}_{k=0}^{n-1}$ in $\vec{D}$. Buckholtz [1] proved that

$$
W^{-1}=\lim _{n \rightarrow \infty} H_{n}^{1 / n}=\sup _{1 \leq n<\infty} H_{n}^{1 / n},
$$

and that there exists a constant $\beta, 0<\beta<1$, such that

$$
W^{n} H_{n} \geq \beta
$$

for $0 \leq n<\infty$ [3]. From (1.6) the numerical value of $W$ can be (theoretically) calculated to any desired accuracy.

2. Preliminaries. The bound $C_{\lambda} \geq .536$ is a consequence of the following expansion theorem of Pommiez.

Theorem. Suppose that $f$ is analytic in $D$ and $c$ is a number such that $0 \leq$ $c \leq .536$. Let $\left\{z_{n}\right\}_{n=0}^{\infty}$ be a sequence of complex numbers in $|z| \leq c$ and let $\lambda \epsilon$ $c_{0}$. Then there exists a sequence $\left\{C_{n}\right\}$ of polynomials, $C_{n}$ of degree $n$, such that for all $z \in D$,

$$
f(z)=f\left(z_{0}\right)+\sum_{n=1}^{\infty} \Delta_{\lambda}^{n} f\left(z_{n}\right) C_{n}(z) .
$$

From (2.1) the conditions $\Delta_{\lambda}^{n} f\left(z_{n}\right)=0,0 \leq n<\infty$, imply $f \equiv 0$. Thus $C_{\boldsymbol{\lambda}} \geq$ 536, $\lambda \in c_{0}$.

We seek an expansion of the form (2.1) with milder restrictions on the sequence $\left\{z_{k}\right\}$. Applying (2.1) to $F(z)=\left(z-\lambda_{1}\right)\left(z-\lambda_{2}\right) \cdots\left(z-\lambda_{n}\right)$ and noting $\Delta^{k} F(z)=$ $\left(z-\lambda_{k+1}\right)\left(z-\lambda_{k+2}\right) \cdots\left(z-\lambda_{n}\right), 0 \leq k \leq n-1$, and $\Delta^{n} F(z)=1$, it follows that the polynomials $C_{n}(z)$ must satisfy

$$
\left(z-\lambda_{1}\right)\left(z-\lambda_{2}\right) \cdots\left(z-\lambda_{n}\right)=\left(z_{0}-\lambda_{1}\right)\left(z_{0}-\lambda_{2}\right) \cdots\left(z_{0}-\lambda_{n}\right)
$$

$$
+\sum_{k=1}^{n-1}\left(z_{k}-\lambda_{k+1}\right) \cdots\left(z_{k}-\lambda_{n}\right) C_{k}(z)+C_{n}(z)
$$


Then clearly $C_{n}(z)$ depends on $z_{0}, z_{1}, \ldots, z_{n-1}$ and $\lambda_{1}, \lambda_{2}, \ldots, \lambda_{n}$. Taking $C_{0}(z)=1,(2.2)$ becomes

$$
\begin{aligned}
C_{n}\left(z ; z_{0}, z_{1}, \ldots, z_{n-1} ; \lambda_{1}, \lambda_{2}, \ldots, \lambda_{n}\right) \\
=\left(z-\lambda_{1}\right) \ldots\left(z-\lambda_{n}\right)-\sum_{k=0}^{n-1}\left(z_{k}-\lambda_{k+1}\right) \ldots\left(z_{k}-\lambda_{n}\right) \\
\quad \quad C_{k}\left(z ; z_{0}, z_{1}, \ldots, z_{k-1} ; \lambda_{1}, \lambda_{2}, \ldots, \lambda_{k}\right) .
\end{aligned}
$$

We therefore take (2.3) as our recursive defining relation, without regard to the restrictions on $\left\{z_{k}\right\}_{k=0}^{n-1}$ imposed by Pommiez's theorem.

Lemma 1. Let $\left\{z_{j}\right\}_{j=0}^{\infty}$ and $\left\{\lambda_{j}\right\}_{j=1}^{\infty}$ be sequences of complex numbers. The following identities bold:

$$
\begin{aligned}
& \Delta{ }^{k} C_{n}\left(z ; z_{0}, \ldots, z_{n-1} ; \lambda_{1}, \ldots, \lambda_{n}\right) \\
& =C_{n-k}\left(z ; z_{k}, \ldots, z_{n-1} ; \lambda_{k+1}, \ldots, \lambda_{n}\right), \quad \text { for } 0 \leq k \leq n, \\
& C_{n}\left(z_{0} ; z_{0}, \ldots, z_{n-1} ; \lambda_{1}, \ldots, \lambda_{n}\right)=0, \quad n \geq 1, \\
& \Delta^{k} C_{n}\left(z_{k} ; z_{0}, \ldots, z_{n-1} ; \lambda_{1}, \ldots, \lambda_{n}\right)=\delta_{k n}, \quad 0 \leq k, n<\infty,
\end{aligned}
$$

where $\delta_{k n}$ denotes the Kronecker delta,

$$
\begin{aligned}
& C_{n}\left(\alpha z ; \alpha z_{0}, \ldots, \alpha z_{n-1} ; \alpha \lambda_{1}, \ldots, \alpha \lambda_{n}\right) \\
& \quad=\alpha^{n} C_{n}\left(z ; z_{0}, \ldots, z_{n-1} ; \lambda_{1}, \ldots, \lambda_{n}\right), \quad n \geq 0,
\end{aligned}
$$

where $\alpha$ is a complex number.

$C_{n}\left(z ; z_{0}, \ldots, z_{n-1} ; \lambda_{1}, \ldots, \lambda_{n}\right)$

$$
\begin{aligned}
& =\sum_{k=0}^{n} C_{n-k}\left(\lambda_{k+1} ; z_{k}, \ldots, z_{n-1} ; \lambda_{k+1}, \ldots, \lambda_{n}\right)\left(z-\lambda_{1}\right)\left(z-\lambda_{2}\right) \cdots\left(z-\lambda_{k}\right), \\
& n \geq 0 \\
& C_{n}\left(z ; z_{0}, \ldots, z_{n-1} ; 0, \ldots, 0\right)=B_{n}\left(z ; z_{0}, \cdots, z_{n-1}\right), \quad n \geq 0 .
\end{aligned}
$$

Proof. In (2.8), take $\left(z-\lambda_{1}\right) \cdots\left(z-\lambda_{k}\right)$ to be 1 if $k=0$. Note that (2.6) implies that the polynomials $C_{n}(z), 0 \leq n<\infty$, together with the sequence of linear functionals $f \rightarrow \Delta^{k} f\left(z_{k}\right)^{n}, 0 \leq k<\infty$, form a biorthonormal system [4].

We establish (2.4) by induction on $n$. If $n=0$, then $k=0$ and the result is trivial. Let $m$ be a positive integer and suppose that for each $j$ such that $0 \leq j \leq m$. -1 we have $\Delta^{k} C_{j}\left(z ; z_{0}, \ldots, z_{j-1} ; \lambda_{1}, \ldots, \lambda_{j}\right)=C_{j-k}\left(z ; z_{k}, \ldots, z_{j-1} ; \lambda_{k+1}, \ldots, \lambda_{j}\right)$, 
$0 \leq k \leq j$. Note that $k>j$ implies $\Delta^{k} C_{j}(z)=0$, since $C_{j}(z)$ is a polynomial of degree $j$ in $z$. By (2.3) and the induction hypothesis, $k \leq m-1$ implies

$$
\begin{aligned}
\Delta^{k} C_{m} & \left(z ; z_{0}, \ldots, z_{m-1} ; \lambda_{1}, \ldots, \lambda_{m}\right) \\
= & \Delta^{k}\left(z-\lambda_{1}\right) \ldots\left(z-\lambda_{m}\right) \\
& \quad-\sum_{j=0}^{m-1}\left(z_{j}-\lambda_{j+1}\right) \ldots\left(z_{j}-\lambda_{m}\right) \Delta^{k} C_{j}\left(z ; z_{0}, \ldots, z_{j-1} ; \lambda_{1}, \ldots, \lambda_{j}\right) \\
= & \left(z-\lambda_{k+1}\right) \ldots\left(z-\lambda_{m}\right) \\
& -\sum_{j=k}^{m-1}\left(z_{j}-\lambda_{j+1}\right) \ldots\left(z_{j}-\lambda_{m}\right) C_{j-k}\left(z ; z_{k}, \ldots, z_{j-1} ; \lambda_{k+1}, \ldots, \lambda_{j}\right) \\
= & \left(z-\lambda_{k+1}\right) \ldots\left(z-\lambda_{m}\right) \\
& \quad-\sum_{p=0}^{m-k-1}\left(z_{p+k}-\lambda_{p+k+1}\right) \ldots\left(z_{p+k}-\lambda_{m}\right) C_{p}\left(z ; z_{k}, \ldots, z_{p+k-1} ; \lambda_{k+1}, \ldots, \lambda_{p+k}\right) \\
= & C_{m-k}\left(z ; z_{k}, \ldots, z_{m-1} ; \lambda_{k+1}, \ldots, \lambda_{m}\right) .
\end{aligned}
$$

Since (2.3) implies $\Delta^{m} C_{m}\left(z ; z_{0}, \ldots, z_{m-1} ; \lambda_{1}, \ldots, \lambda_{m}\right)=\Delta^{m}\left(z-\lambda_{1}\right) \ldots$ $\left(z-\lambda_{m}\right)=1$, the proof of (2.4) is complete.

For the proof of (2.5), note first that $C_{1}\left(z_{0} ; z_{0} ; \lambda_{1}\right)=\left(z_{0}-\lambda_{1}\right)-$ $\left(z_{0}-\lambda_{1}\right) C_{0}\left(z_{0}\right)=0$. Let $m \geq 2$ be an integer and suppose

$$
C_{j}\left(z_{0} ; z_{0}, \ldots, z_{j-1} ; \lambda_{1}, \ldots, \lambda_{j}\right)=0 \quad \text { for } 1 \leq j \leq m-1 .
$$

Then

$$
\begin{aligned}
C_{m}\left(z_{0} ; z_{0}, \ldots, z_{m-1} ; \lambda_{1}, \ldots, \lambda_{m}{ }^{\prime}\right. & \\
= & \left(z_{0}-\lambda_{1}\right) \ldots\left(z_{0}-\lambda_{m}\right) \\
& \quad-\sum_{j=0}^{m-1}\left(z_{j}-\lambda_{j+1}\right) \ldots\left(z_{j}-\lambda_{m}\right) C_{j}\left(z_{0} ; z_{0}, \ldots, z_{j-1} ; \lambda_{1}, \ldots, \lambda_{j}\right) \\
= & \left(z_{0}-\lambda_{1}\right) \ldots\left(z_{0}-\lambda_{m}\right)-\left(z_{0}-\lambda_{1}\right) \ldots\left(z_{0}-\lambda_{m}\right)=0 .
\end{aligned}
$$

The proofs of (2.7) and (2.9) are similar. Equation (2.6) follows from (2.4) and (2.5), together with the fact that $C_{n}(z)$ is a polynomial of degree $n$ in $z$; (2.8) is the Newton series expansion of $C_{n}\left(z ; z_{0}, \ldots, z_{n-1} ; \lambda_{1}, \ldots, \lambda_{n}\right)$. A convenient representation for the coefficient $C_{n}\left(\lambda_{1} ; z_{0}, \ldots, z_{n-1}\right.$; $\left.\lambda_{1}, \ldots, \lambda_{n}\right)$ in (2.8) is obtained by considering the infinite upper triangular matrices $A$ and $B$ defined as follows: for $0 \leq j, k<\infty$ let 


$$
A_{j k}=\left\{\begin{array}{l}
C_{k-j}\left(\lambda_{j+1} ; z_{j}, \ldots, z_{k-1} ; \lambda_{j+1}, \ldots, \lambda_{k}\right), \quad j<k \\
1, \quad j=k, \\
0, \quad j>k
\end{array}\right.
$$

$$
B_{j k}= \begin{cases}\left(z_{j}-\lambda_{j+1}\right)\left(z_{j}-\lambda_{j+2}\right) \cdots\left(z_{j}-\lambda_{k}\right), \quad j<k, \\ 1, \quad j=k, \\ 0, \quad j>k .\end{cases}
$$

Thus $A_{k n}$ is the $k$ th coefficient of $C_{n}\left(z ; z_{0}, \ldots, z_{n-1} ; \lambda_{1}, \ldots, \lambda_{n}\right)$ in the expansion (2.8). It is easy to show that the matrices $A$ and $B$ are mutually reciprocal. For $j<n,(2.3)$ implies

$$
\begin{aligned}
& \sum_{k=j}^{n} A_{j k} B_{k n}=\sum_{k=j}^{n} C_{k-j}\left(\lambda_{j+1} ; z_{j}, \ldots, z_{k-1} ; \lambda_{j+1}, \ldots, \lambda_{k}\right)\left(z_{k}-\lambda_{k+1}\right) \cdots\left(z_{k}-\lambda_{n}\right) \\
& \quad=\sum_{m=0}^{n-j} C_{m}\left(\lambda_{j+1} ; z_{j}, \cdots, z_{m+j-1} ; \lambda_{j+1}, \ldots, \lambda_{m+j}\right)\left(z_{m+j}-\lambda_{m+j+1}\right) \cdots\left(z_{m+j}-\lambda_{n}\right) \\
& \quad=\left(\lambda_{j+1}-\lambda_{j+1}\right)\left(\lambda_{j+1}-\lambda_{j+2}\right) \ldots\left(\lambda_{j+1}-\lambda_{n}\right)=0 .
\end{aligned}
$$

By applying (2.8) and then (2.6) and (2.4), we obtain

$$
\begin{aligned}
\sum_{k=j}^{n} B_{j k} A_{k n}= & \sum_{k=j}^{n}\left(z_{j}-\lambda_{j+1}\right) \cdots\left(z_{j}-\lambda_{k}\right) C_{n-k}\left(\lambda_{k+1} ; z_{k}, \ldots, z_{n-1} ; \lambda_{k+1}, \ldots, \lambda_{n}\right) \\
=\sum_{m=0}^{n-j}\left(z_{j}-\lambda_{j+1}\right) & \cdots\left(z_{j}-\lambda_{j+m}\right) \\
& \cdot C_{n-j-m}\left(\lambda_{m+j+1} ; z_{m+j}, \cdots, z_{n-1} ; \lambda_{m+j+1}, \ldots, \lambda_{n}\right) \\
= & C_{n-j}\left(z_{j} ; z_{j}, \ldots, z_{n-1} ; \lambda_{j+1}, \ldots, \lambda_{n}\right)=0 .
\end{aligned}
$$

Since $A_{n n}=B_{n n}=1,0 \leq n<\infty$, It follows that $A B=B A=I$, where $I$ is the identity matrix.

Let $m$ be a nonnegative integer and define the $(m+1)$ by $(m+1)$ matrices $A^{m}$ and $B^{m}$ by

$$
A_{j k}^{m}=A_{j k}, \quad B_{j k}^{m}=B_{j k}, \quad 0 \leq j, k \leq m .
$$


Arguing as above, it follows that $A^{m}$ and $B^{m}$ are inverses. Hence the entries in $A^{m}$ can be determined by considering the cofactors in $B^{m}$. In particular, (2.11)

$$
\begin{aligned}
& A_{0, m}^{m}=C_{m}\left(\lambda_{1} ; z_{0}, \ldots, z_{m-1} ; \lambda_{1}, \ldots, \lambda_{m}\right) \\
& =(-1)^{m} \operatorname{Det}\left[\begin{array}{cccc}
\left(z_{0}-\lambda_{1}\right) & \left(z_{0}-\lambda_{1}\right)\left(z_{0}-\lambda_{2}\right) & \ldots & {\left[\left(z_{0}-\lambda_{1}\right)\left(z_{0}-\lambda_{2}\right) \cdots\left(z_{0}-\lambda_{m}\right)\right]} \\
1 & \left(z_{1}-\lambda_{2}\right) & \ldots & {\left[\left(z_{1}-\lambda_{2}\right)\left(z_{1}-\lambda_{3}\right) \cdots\left(z_{1}-\lambda_{m}\right)\right]} \\
0 & 1 & \cdots & {\left[\left(z_{2}-\lambda_{3}\right)\left(z_{2}-\lambda_{4}\right) \cdots\left(z_{2}-\lambda_{m}\right)\right]} \\
\cdot & \cdot & & \cdot \\
\cdot & \cdot & & \cdot \\
\cdot & \cdot & & \cdot \\
0 & 0 & \cdots & \left(z_{m-1}-\lambda_{m}\right)
\end{array}\right] .
\end{aligned}
$$

We will use (2.11) in establishing a relationship, in analogy to (2.9), between $C_{n}\left(\lambda_{1} ; z_{0}, \cdots, z_{n-1} ; \lambda_{1}, \ldots, \lambda_{n}\right)$ and $B_{n}\left(0 ; z_{0}, \ldots, z_{n-1}\right)$. Note first that if $z_{0} \neq 0$, then (2.3) and (1.5) give $C_{1}\left(\lambda_{1} ; z_{0} ; \lambda_{1}\right)=-\left(z_{0}-\lambda_{1}\right)=$ $\left(\left(z_{0}-\lambda_{1}\right) / z_{0}\right)\left(-z_{0}\right)=\left(\left(z_{0}-\lambda_{1}\right) / z_{0}\right) B_{1}\left(0 ; z_{0}\right)$. Substituting this expression in to (2.3), with $n=2$, and using (1.5), we obtain

$$
C_{2}\left(\lambda_{1} ; z_{0}, z_{1} ; \lambda_{1}, \lambda_{2}\right)=\left(\left(z_{0}-\lambda_{1}\right) / z_{0}\right) B_{2}\left(Q ; z_{0}, z_{1}\right),
$$

and similarly,

$C_{3}\left(\lambda_{1} ; z_{0}, z_{1}, z_{2} ; \lambda_{1}, \lambda_{2}, \lambda_{3}\right)=\left(\left(z_{0}-\lambda_{1}\right) ; z_{0}\right)\left[B_{3}\left(0 ; z_{0}, z_{1}, z_{2}\right)-\lambda_{2} B_{2}\left(0 ; z_{0}, z_{1}\right)\right]$.

In general, we can prove the following result.

Theorem 2. If $n \geq 2$ and $z_{0}, z_{1}, \cdots, z_{n-1}$ are nonzero, then

$$
\begin{gathered}
C_{n}\left(\lambda_{1} ; z_{0}, z_{1}, \ldots, z_{n-1} ; \lambda_{1}, \lambda_{2}, \ldots, \lambda_{n}\right) \\
=\left(\frac{z_{0}-\lambda_{1}}{z_{0}}\right)_{\substack{2 \leq j_{1}<\cdots<j_{k} \leq n-1 \\
0 \leq k<n-2}}(-1)^{k} \lambda_{j_{1}} \lambda_{j_{2}} \cdots \lambda_{j_{k}} \\
\cdot \sum_{n-k}\left(0 ; z_{0}, z_{1}, \ldots, \widehat{z_{j_{1}}}, \cdots, \widehat{z_{j_{2}}}, \ldots, \widehat{z_{j_{k}}}, \cdots, z_{n-1}\right) .
\end{gathered}
$$

The summation in (2.12) is taken over all possible configurations $\lambda_{p_{1}} \lambda_{p_{2}}$ $\cdots \lambda_{p_{k}}$ such that $2 \leq p_{1}<p_{2}<\cdots<p_{k} \leq n-1$ and $0 \leq k \leq n-2$; if $k=0$ we take $\lambda_{p_{1}} \ldots \lambda_{p_{k}}=1$. The symbol $\widehat{z_{j_{i}}}$ means that the variable $z_{j_{i}}$ has been removed; thus 
376

G. W. CROFTS AND J. K. SHAW

$$
\begin{gathered}
B_{n-k}\left(0 ; z_{0}, z_{1}, \ldots, \widehat{z_{j_{1}}}, \ldots, \widehat{z_{j_{2}}}, \ldots, \widehat{z_{j_{k}}}, \ldots, z_{n-1}\right) \\
=B_{n-k}\left(0 ; z_{0}, z_{1}, \ldots, z_{j_{1}-1}, z_{j_{1+1}}, \ldots, z_{j_{2}-1},\right. \\
\left.z_{j_{2}+1}, \cdots, z_{j_{k}-1}, z_{j_{k}+1}, \ldots, z_{n-1}\right),
\end{gathered}
$$

To prove Theorem 2, we need the following technical lemma concerning the remainder polynomials.

Lemma 2. If $n \geq 1$, then

$$
\begin{aligned}
& B_{n+1}\left(0 ; z_{0}, z_{1}, z_{2}, \ldots, z_{n}\right) \\
& \quad=z_{0} B_{n}\left(0 ; z_{0}, z_{2}, z_{3}, \ldots, z_{n}\right)-z_{0} B_{n}\left(0 ; z_{1}, z_{2}, \ldots, z_{n}\right) .
\end{aligned}
$$

If $n \geq 2, z_{0} \neq 0$ and $z_{1} \neq 0$, then

$$
\begin{aligned}
& B_{n}\left(0 ; z_{0}, z_{1}, z_{3}, z_{4}, \ldots, z_{n}\right) \\
& \quad=B_{n}\left(0 ; z_{0}, z_{2}, z_{3}, \ldots, z_{n}\right)-\left(z_{0} / z_{1}\right) B_{n}\left(0 ; z_{1}, z_{2}, \ldots, z_{n}\right) .
\end{aligned}
$$

Proof. Consider (2.13). If $n=1$, then

$$
z_{0} B_{1}\left(0 ; z_{0}\right)-z_{0} B_{1}\left(0 ; z_{1}\right)=z_{0}\left(-z_{0}\right)-z_{0}\left(-z_{1}\right)=-z_{0}^{2}+z_{0} z_{1}=B_{2}\left(0 ; z_{0}, z_{1}\right) \text {. }
$$

Let $n$ be a positive integer and suppose that (2.13) holds for the integers $k$ such that $1 \leq k \leq n$. Then by (1.5),

$$
\begin{aligned}
& B_{n+2}\left(0 ; z_{0}, z_{1}, z_{2}, \ldots, z_{n+1}\right)=-\sum_{k=0}^{n+1} z_{k}^{n+2-k} B_{k}\left(0 ; z_{0}, z_{1}, \ldots, z_{k-1}\right) \\
& =-z_{0}^{n+2}-z_{1}^{n+1} B_{1}\left(0 ; z_{0}\right)-\sum_{k=2}^{n+1} z_{k}^{n+2-k_{2}} z_{0} B_{k-1}\left(0 ; z_{0}, z_{2}, \ldots, z_{k-1}\right) \\
& \left.\quad-B_{k-1}\left(0 ; z_{1}, z_{2}, \ldots, z_{k-1}\right)\right] \\
& =-z_{0}^{n+2}-z_{0} \sum_{k=2}^{n+1} z_{k}^{n+2-k} B_{k-1}\left(0 ; z_{0}, z_{2}, \ldots, z_{k-1}\right) \\
& \quad+z_{0} z_{1}^{n+1}+z_{0} \sum_{k=2}^{n+1} z_{k}^{n+2-k} B_{k-1}\left(0 ; z_{1}, z_{2}, \ldots, z_{k-1}\right) \\
& =z_{0} B_{n+1}\left(0 ; z_{0}, z_{2}, z_{3}, \ldots, z_{n+1}\right)-z_{0} B_{n+1}\left(0 ; z_{1}, z_{2}, \ldots, z_{n+1}\right) .
\end{aligned}
$$


For the proof of (2.14) note first that $B_{2}\left(0 ; z_{0}, z_{2}\right)-\left(z_{0} / z_{1}\right) B_{2}\left(0 ; z_{1}, z_{2}\right)=$ $-z_{0}^{2}+z_{0} z_{2}-\left(z_{0} / z_{1}\right)\left(-z_{1}^{2}+z_{1} z_{2}\right)=-z_{0}^{2}+z_{0} z_{2}+z_{0} z_{1}-z_{0} z_{2}=-z_{0}^{2}+$ $z_{0} z_{1}=B_{2}\left(0 ; z_{0}, z_{1}\right)$. If $(2.14)$ holds for the integers $k$ such that $2 \leq k \leq n$, then

$$
\begin{gathered}
B_{n+1}\left(0 ; z_{0}, z_{1}, z_{3}, \ldots, z_{n+1}\right) \\
=-z_{0}^{n+1}-z_{1}^{n}\left(-z_{0}\right)-\sum_{k=3}^{n+1} z_{k}^{n+2-k} B_{k-1}\left(0 ; z_{0}, z_{1}, z_{3}, \ldots, z_{k-1}\right) \\
=-z_{0}^{n+1}+z_{0} z_{1}^{n}-\sum_{k=3}^{n+1} z_{k}^{n+2-k}\left[B_{k-1}\left(0 ; z_{0}, z_{2}, z_{3}, \ldots, z_{k-1}\right)\right. \\
\left.\quad-\frac{z_{0}}{z_{1}} B_{k-1}\left(0 ; z_{1}, z_{2}, \ldots, z_{k-1}\right)\right] \\
=-z_{0}^{n+1}-z_{2}^{n} B_{1}\left(0 ; z_{0}\right)-\sum_{k=3}^{n+1} z_{k}^{n+2-k_{k-1}}\left(0 ; z_{0}, z_{2}, \ldots, z_{k-1}\right)
\end{gathered}
$$$$
+z_{0} z_{1}^{n}+z_{2}^{n} B_{1}\left(0 ; z_{0}\right)+\frac{z_{0}}{z_{1}} \sum_{k=3}^{n+1} z_{k}^{n+2-k} B_{k-1}\left(0 ; z_{1}, z_{2}, \ldots, z_{k-1}\right)
$$$$
=-z_{0}^{n+1}-\sum_{k=2}^{n+1} z_{k}^{n+2-k} B_{k-1}\left(0 ; z_{0}, z_{2}, z_{3}, \ldots, z_{k-1}\right)
$$$$
+\frac{z_{0}}{z_{1}}\left[z_{1}^{n+1}+z_{2}^{n} B_{1}\left(0 ; z_{0}\right) \frac{z_{1}}{z_{0}}+\sum_{k=3}^{n+1} z_{k}^{n+2-k} B_{k-1}\left(0 ; z_{1}, z_{2}, \ldots, z_{k-1}\right)\right]
$$$$
=B_{n+1}\left(0 ; z_{0}, z_{2}, z_{3}, \ldots, z_{n+1}\right)
$$$$
+\frac{z_{0}}{z_{1}}\left[z_{1}^{n+1}+z_{2}^{n} B_{1}\left(0 ; z_{1}\right)+\sum_{k=3}^{n+1} z_{k}^{n+2-k} B_{k-1}\left(0 ; z_{1}, z_{2}, \ldots, z_{k-1}\right)\right]
$$$$
=B_{n+1}\left(0 ; z_{0}, z_{2}, \ldots, z_{n+1}\right)-\left(z_{0} / z_{1}\right) B_{n+1}\left(0 ; z_{1}, z_{2}, \ldots, z_{n+1}\right) \text {, }
$$

and this completes the proof.

Proof of Theorem 2. The proof is by induction; the case $n=2$ has been established. Suppose now that $n>2$ and (2.12) holds for the integers $k$ such that $2 \leq k \leq n-1$. Taking $m=n$ in (2.11) and expanding the determinant by the first column, we have

$$
\begin{aligned}
& C_{n}\left(\lambda_{1} ; z_{0}, \ldots, z_{n-1} ; \lambda_{1}, \ldots, \lambda_{n}\right) \\
& =(-1)^{n}\left[(-1)^{n-1}\left(z_{0}-\lambda_{1}\right) C_{n-1}\left(\lambda_{2} ; z_{1}, z_{2}, \ldots, z_{n-1} ; \lambda_{2}, \lambda_{3}, \ldots, \lambda_{n}\right)\right] \\
& \quad-(-1)^{n}\left[(-1)^{n-1}\left(z_{0}-\lambda_{2}\right) C_{n-1}\left(\lambda_{1} ; z_{0}, z_{2}, \ldots, z_{n-1} ; \lambda_{1}, \lambda_{3}, \ldots, \lambda_{n}\right)\right] .
\end{aligned}
$$

By the induction hypothesis, 
378

G. W. CROFTS AND J. K. SHAW

$$
\begin{aligned}
& C_{n}\left(\lambda_{1} ; z_{0}, \cdots, z_{n-1} ; \lambda_{1}, \ldots, \lambda_{n}\right) \\
& =-\left(z_{0}-\lambda_{1}\right)\left(\frac{z_{1}-\lambda_{2}}{z_{1}}\right) \\
& \sum_{3 \leq i_{1}<\cdots<j_{k} \leq n-1}(-1)^{k} \lambda_{j_{1}} \cdots \lambda_{j_{k}} B_{n-k-1}\left(0 ; z_{1}, z_{2}, \ldots, \widehat{z_{j_{1}}}, \ldots,{\widehat{z_{j}}}_{j_{k}}, \cdots, z_{n-1}\right) \\
& 0 \leq k \leq n-3 \\
& +\left(z_{0}-\lambda_{2}\right)\left(\frac{z_{0}-\lambda_{1}}{z_{0}}\right) \\
& \sum_{3 \leq j_{1}<\cdots<j_{k} \leq n-1}(-1)^{k} \lambda_{j_{1}} \cdots \lambda_{j_{k}} B_{n-k-1}\left(0 ; z_{0}, z_{2}, \ldots, \widehat{z_{j_{1}}}, \ldots, \widehat{z_{j_{k}}}, \cdots, z_{n-1}\right) . \\
& 0 \leq k \leq n-3
\end{aligned}
$$

By (2.13) and (2.14), the coefficient of $\left(\left(z_{0}-\lambda_{1}\right) / z_{0}\right)(-1)^{k} \lambda_{j_{1}} \cdots \lambda_{j_{k}}$ is given by

$$
\begin{aligned}
& -\left(\left(z_{1}-\lambda_{2}\right) / z_{1}\right) z_{0} B_{n-k-1}\left(0 ; z_{1}, z_{2}, \ldots, \widehat{z_{j_{1}}}, \ldots, \widehat{z_{j_{k}}}, \ldots, z_{n-1}\right) \\
& +\left(z_{0}-\lambda_{2}\right) B_{n-k-1}\left(0 ; z_{0}, z_{2}, \ldots, \widehat{z_{j_{1}}}, \ldots, \widehat{z_{j_{k}}}, \ldots, z_{n-1}\right) \\
& =z_{0} B_{n-k-1}\left(0 ; z_{0}, z_{2}, \ldots, \widehat{z_{j_{1}}}, \ldots, \widehat{z_{j_{k}}}, \ldots, z_{n-1}\right) \\
& -z_{0} B_{n-k-1}\left(0 ; z_{1}, z_{2}, \ldots, \widehat{z_{j_{1}}}, \ldots, \widehat{z_{j}}, \ldots, z_{n-1}\right) \\
& -\lambda_{2}\left[B_{n-k-1}(0 ; z_{0}, z_{2}, \ldots, \overbrace{j_{1}}^{1}, \ldots, \overbrace{z_{k}}^{\gamma_{2}}, \ldots, z_{n-1})\right. \\
& \left.-\left(z_{0} / z_{1}\right) B_{n-k-1}\left(0 ; z_{1}, z_{2}, \ldots, \widehat{z_{j_{1}}}, \ldots, \widehat{z_{j_{k}}}, \ldots, z_{n-1}\right)\right] \\
& =B_{n-k}\left(0 ; z_{0}, z_{1}, z_{2}, \ldots, \widehat{z_{j_{1}}}, \ldots, \widehat{z_{j_{k}}}, \ldots, z_{n-1}\right) \\
& -\lambda_{2} B_{n-k-1}\left(0 ; z_{0}, z_{1}, \widehat{z_{2}}, \ldots, \widehat{z}_{j_{1}}, \ldots, \widehat{z_{j_{k}}}, \ldots, z_{n-1}\right) \text {. }
\end{aligned}
$$

Hence,

$$
\begin{aligned}
& C_{n}\left(\lambda_{1} ; z_{0}, \ldots, z_{n-1} ; \lambda_{1}, \ldots, \lambda_{n}\right) \\
& =\left(\frac{z_{0}-\lambda_{1}}{z_{0}}\right)_{\substack{3 \leq j_{1}<\cdots<j_{k} \leq n-1 \\
0 \leq k \leq n-3}}(-1)^{k} \lambda_{j_{1}} \ldots \lambda_{j_{k}} \\
& +\left(\frac{z_{0}-\lambda_{1}}{z_{0}}\right)_{\substack{3 \leq j_{1}<\cdots<j_{k} \leq n-1 \\
0 \leq k \leq n-3}}(-1)^{k+1} \lambda_{2} \lambda_{j_{1}} \ldots \lambda_{j_{k}} B_{B_{n-k}}\left(0 ; z_{0}, z_{1}, \ldots, \widehat{z_{j}}, \ldots, \widehat{z_{j}}, \ldots, z_{n-1}\right) \\
& \cdot B_{n-k-1}\left(0 ; z_{0}, z_{1}, \widehat{z_{2}}, \ldots, \widehat{z_{j_{1}}}, \ldots, \widehat{z_{j}}, \ldots, z_{n-1}\right)
\end{aligned}
$$




$$
\begin{aligned}
& =\left(\frac{z_{0}-\lambda_{1}}{z_{0}}\right)_{\substack{2 \leq j_{1}<\cdots<j_{k} \leq n-1 \\
0 \leq k \leq n-2}}(-1)^{k} \lambda_{j_{1}} \cdots \lambda_{j_{k}} \\
& \cdot B_{n-k}\left(0 ; z_{0}, z_{1}, \ldots, \widehat{z_{j_{1}}}, \ldots, \widehat{z_{j k}}, \cdots, z_{n-1}\right),
\end{aligned}
$$

and this completes the proof.

We conclude this section now with a lemma to be used in $\$ 3$ below.

Lemma 3. Let $n \geq 1$ and suppose that the complex numbers $z_{0}, z_{1}, \cdots, z_{n-1}$ lie in the disc $|z| \leq W$. Then

$$
\left|B_{n}\left(0 ; z_{0}, \cdots, z_{n-1}\right)\right| \leq 1 .
$$

Moreover, there exist complex numbers $\zeta_{0}, \zeta_{1}, \ldots, \zeta_{n-1}$ on the circle $|z|=W$ sucb that

$$
\left|B_{n}\left(0 ; \zeta_{0}, \zeta_{1}, \ldots, \zeta_{n-1}\right)\right|=W^{n} H_{n} \text {. }
$$

Proof. Let $w_{k}=z_{k} / W, 0 \leq k \leq n-1$. Then $\left|w_{k}\right| \leq 1,0 \leq k \leq n-1$, and (2.7), (2.9) and (1.6) imply $\left|B_{n}\left(0 ; z_{0}, \ldots, z_{n-1}\right)\right|=W^{n}\left|B_{n}\left(0 ; w_{0}, \ldots, w_{n-1}\right)\right|$ $\leq W^{n} H_{n} \leq 1$. For (2.16), the maximum principle permits us to choose points $w_{0}^{\prime}$, $w_{1}^{\prime}, \ldots, w_{n-1}^{\prime}$ on the circle $|z|=1$ such that $\left|B_{n}\left(0 ; w_{0}^{\prime}, \ldots, w_{n-1}^{\prime}\right)\right|=H_{n}$. If $\zeta_{k}=W w_{k}^{\prime}, 0 \leq k \leq n-1$, then $\left|B_{n}\left(0 ; \zeta_{0}, \ldots, \zeta_{n-1}\right)\right|=\left|W^{n} B_{n}\left(0 ; w_{0}^{\prime}, \ldots, w_{n-1}^{\prime}\right)\right|$ $=W^{n} H_{n}$, and this completes the proof.

3. Matrix transformations on the space $\mathbb{G}_{r}$. If $r>0$, we denote by $\mathbb{G}_{r}$ the complex vector space of functions analytic in the disc $D_{r}=\{z:|z|<r\} ; \mathbb{G}_{r}^{r}$, given the topology of uniform convergence on compact subsets of $D_{r}$, is known to be a Fréchet-Montel space [8].

Let $\lambda \in c_{0}$ have terms in $D_{r}$ and define $\sigma_{0}(z)=1, \sigma_{n}(z)=\left(z-\lambda_{1}\right)\left(z-\lambda_{2}\right)$ $\ldots\left(z-\lambda_{n}\right), n=1,2,3, \ldots$ Then $\left\{\sigma_{n}\right\}_{n=0}^{\infty}$ is a basis for $\mathbb{G}_{r}[10]$; in fact, $f=$ $\sum_{n=0}^{\infty} a_{n} \sigma_{n}$ is the Newton series expansion for $f \in \mathbb{G}_{r}$. It is not difficult to show that $f=\sum_{n=0}^{\infty} a_{n} \sigma_{n}$ belongs to $\mathcal{G}_{r}$ if and only if $\lim \sup _{n \rightarrow \infty}\left|a_{n}\right|^{1, n} \leq r^{-1}$. Thus we may identify $\mathfrak{G}_{r}$ with the space of complex sequences $x=\left(x_{0}, x_{1}, \ldots\right)$ such that $\lim \sup _{n \rightarrow \infty}\left|x_{n}\right|^{1 / n} \leq 1 / r$.

Let $M$ be an infinite complex matrix and let $f=\left(f_{0}, f_{1}, \ldots\right) \in \mathbb{A}_{r}$. Then $M f$ is the sequence whose $n$th term is given by $(M f)_{n}=\sum_{k=0}^{\infty} M_{n k} f_{k}$. We say that $M$ maps $\mathbb{G}_{r}$ to $\mathbb{G}_{r}$ provided that $f \in \mathbb{G}_{r}$ implies $M f \in \mathbb{G}_{r}$. The following theorem of M. M. Dragilev $([5],[6])$ characterizes a large class of matrix transformations on $\hat{4}_{r}$.

Theorem 3. Let $M$ be an infinite upper triangular matrix such that $M_{k k}=$ $1,0 \leq k<\infty$, let $N$ denote the unique upper triangular inverse of $M$, and suppose 
$R>0$. A necessary and sufficient condition that either $M$ or $N$ map $\mathbb{G}_{r}$ one-toone and onto $\mathbb{G}_{r}$ for each $r>R$ is that

$$
\lim \sup _{n \rightarrow \infty}\left[\max _{0 \leq j \leq n}\left|M_{j n}\right| R^{j-n}\right]^{1 / n} \leq 1
$$

and

$$
\underset{n \rightarrow \infty}{\lim \sup }\left[\max _{0 \leq j \leq n}\left|N_{j n}\right| R^{j-n}\right]^{1 / n} \leq 1
$$

Furthermore, it is not difficult to show that either $M$ and $N$ simultaneously map $\mathscr{G}_{r}$ one-to-one onto itself or neither does.

Lemma 4. Suppose $R>0$ and let $\left\{\lambda_{k}\right\}_{k=1}^{\infty}$ and $\left\{z_{k}\right\}_{k=0}^{\infty}$ be sequences in $D_{R}$ such that $\lambda_{k} \rightarrow 0$ and $\left|z_{k}\right| \leq W R, 0 \leq k<\infty$. Then the matrices $A$ and $B$ of (2.10) satisfy (3.1) and (3.2).

Proof. Consider the matrix B. If $r_{n}=\left|\lambda_{n}\right|, 1 \leq n<\infty$, and $j<k$, then

$$
\begin{aligned}
\left|B_{j k}\right| & =\left|\left(z_{j}-\lambda_{j+1}\right)\left(z_{j}-\lambda_{j+2}\right) \cdots\left(z_{j}-\lambda_{k}\right)\right| \\
& \leq\left(W R+r_{j+1}\right)\left(W R+r_{j+2}\right) \cdots\left(W R+r_{k}\right) \\
& =(W R)^{k-j}\left[\left(1+r_{j+1} / W R\right) \cdots\left(1+r_{k} / W R\right)\right] \\
& \leq(W R)^{k-j}\left(1+\epsilon_{1}\right)\left(1+\epsilon_{2}\right) \cdots\left(1+\epsilon_{k}\right),
\end{aligned}
$$

where $\epsilon_{n}=r_{n} /(W R)$. Now $\epsilon_{n} \rightarrow 0$ and therefore

$$
\lim _{n \rightarrow \infty}\left[\left(1+\epsilon_{1}\right)\left(1+\epsilon_{2}\right) \cdots\left(1+\epsilon_{n}\right)\right]^{1 / n}=1 .
$$

Since $W<1$, we have $\left|B_{j k}\right| R^{j-k} \leq W^{k-j}\left(1+\epsilon_{1}\right)\left(1+\epsilon_{2}\right) \ldots\left(1+\epsilon_{k}\right)<$ $\left(1+\epsilon_{1}\right)\left(1+\epsilon_{2}\right) \ldots\left(1+\epsilon_{k}\right)$ and it follows that

$$
\underset{k \rightarrow \infty}{\lim \sup _{0}}\left[\max _{0 \leq i \leq k}\left|B_{j k}\right| R^{j-k}\right]^{1 / k} \leq 1 .
$$

For the matrix $A,(2.7)$ implies

$$
\left|A_{j k}\right| R^{j-k}=\left|C_{k-j}\left(\lambda_{j+1} / R ; z_{j} / R, \ldots, z_{k-1} / R ; \lambda_{j+1} / R, \ldots, \lambda_{k} / R\right)\right|
$$

for $j<k$. By the maximum principle, there exist points $w_{j}, w_{j+1}, \cdots, w_{k}$ on the circle $|z|=W$ such that $\left|A_{j k}\right| R^{j-k} \leq\left|C_{k-j}\left(\mu_{j+1} ; w_{j}, \cdots, w_{k-1} ; \mu_{j+1}, \cdots, \mu_{k}\right)\right|$, where $\mu_{i}=\lambda_{i} / R, 1 \leq i<\infty$. By (2.12) and Lemma 3 , 


$$
\begin{aligned}
\left|A_{j k}\right| R^{j-k} & \leq\left|\frac{w_{j}-\mu_{j+1}}{w_{j}}\right|_{\substack{j+2 \leq p_{1}<\cdots<p_{n} \leq k-1 \\
0 \leq n \leq k-j-2}}\left|\mu_{p_{1}}\right|\left|\mu_{p_{2}}\right| \cdots\left|\mu_{p_{n}}\right| \\
& \leq \frac{W+1}{W}\left(1+\left|\mu_{1}\right|\right)\left(1+\left|\mu_{2}\right|\right) \cdots\left(1+\left|\mu_{k-1}\right|\right) .
\end{aligned}
$$

Since $\left|\mu_{n}\right| \rightarrow 0$, it follows that $\lim \sup _{k \rightarrow \infty}\left[\max _{0 \leq j \leq k}\left|A_{j k}\right| R^{j-k}\right]^{1 / k} \leq 1$, and this completes the proof.

Theorem 4. Let $f$ be analytic in $D$, let $0<R<1$, and suppose that $\left\{z_{k}\right\}_{k=0}^{\infty}$ and $\left\{\lambda_{k}\right\}_{k=1}^{\infty}$ are sequences in $D$ such that $\left|z_{k}\right| \leq W R, 0 \leq k<\infty$, and $\lambda_{k} \rightarrow 0$. Then

$$
f(z)=\sum_{k=0}^{\infty} \Delta^{k} f\left(z_{k}\right) C_{k}\left(z ; z_{0}, \ldots, z_{k-1} ; \lambda_{1}, \ldots, \lambda_{k}\right),
$$

with uniform convergence on compact subsets of $D$.

Proof. By Theorem 3 and Lemma 4 , the matrices $A$ and $B$ map $\mathfrak{G}_{r}$ one-toone and onto $\mathscr{G}_{r}$ for each $r>R$, where $0<R<1$. Thus $A$ and $B$ map $\mathscr{G}_{1}$ oneto-one onto itself. By a theorem of Köthe and Toeplitz [9], $A$ and $B$ are weakly continuous and, hence, $[11, \mathrm{p} .158]$ are continuous. It follows that $\left\{A\left(\sigma_{n}\right)\right\}_{n=0}^{\infty}$ is a basis for $\mathbb{Q}_{1}$, since $\left\{\sigma_{n}\right\}_{n=0}^{\infty}$ is a basis. But (2.8) implies that $A\left(\sigma_{n}\right)(z)=$ $C_{n}\left(z ; z_{0}, \ldots, z_{n-1} ; \lambda_{1}, \ldots, \lambda_{n}\right)(0 \leq n<\infty)$, and therefore functions $f \in \mathbb{Q}_{1}$ admit expansions $f(z)=\sum_{k=0}^{\infty} \alpha_{k} C_{k}\left(z ; z_{0}, \cdots, z_{k-1} ; \lambda_{1}, \cdots, \lambda_{k}\right)$ with uniform convergence on compact subsets of $D$. The linear functionals $g \rightarrow \Delta^{j} g\left(z_{j}\right), 0 \leq j<\infty$, are readily shown to be continuous. This fact, together with (2.6) implies $a_{k}=$ $\Delta^{k} f\left(z_{k}\right), 0 \leq k<\infty$, which completes the proof.

Since $R<1$ is arbitrary in Theorem 4, (3.3) implies that $C_{\boldsymbol{\lambda}} \geq W$ for all $\lambda \in c_{0}$, which proves the first part of Theorem 1. To complete the proof of Theorem 1 , we will prove that for each $\lambda \in l_{1}$, there exists an extremal function $F$ $\epsilon \mathscr{C}_{1}$ such that each of $\Delta_{\lambda}^{k} F(z)$ has a zero in $|z| \leq W$ but $F \not \equiv 0$. With the expansion (3.3), this will imply $C_{\lambda} \leq W$ for all $\lambda \in l_{1}$.

Thus suppose $\lambda \in l_{1}$ and let $r_{k}=\left|\lambda_{k}\right|, 1 \leq k<\infty$. Then the sequence $s_{n}=$ $\left(1+r_{1}\right)\left(1+r_{2}\right) \ldots\left(1+r_{n}\right)$ converges $[7, \mathrm{p} .223]$ and so there exists a constant $K>1$ such that $\left(1+r_{1}\right)\left(1+r_{2}\right) \ldots\left(1+r_{n}\right)<K$ for all $n$.

Let $N$ be a positive integer such that $r_{n}<W / 2$, for $n \geq N$, and such that $\sum_{n=N}^{\infty} r_{n}<\beta /(\beta+2)$ (see (1.7)). If $n \geq N$, there exist, by Lemma 3, points $z_{0}^{(n)}$, $z_{1}^{(n)}, \ldots, z_{n-1}^{(n)}$ on $|z|=W$ such that

$$
\left|B_{n-N}\left(0 ; z_{N}^{(n)}, z_{N+1}^{(n)}, \cdots, z_{n-1}^{(n)}\right)\right|=W^{n-H_{H}} H_{n-N} .
$$


For $n \geq N$, define $F_{n}(z)=C_{n}\left(z ; z_{0}^{(n)}, \ldots, z_{n-1}^{(n)} ; \lambda_{1}, \ldots, \lambda_{n}\right)$. By $(2.8)$, we have

$$
F_{n}(z)=\sum_{k=0}^{n} C_{n-k}\left(\lambda_{k+1} ; z_{k}^{(n)}, \ldots, z_{n-1}^{(n)} ; \lambda_{k+1}, \cdots, \lambda_{n}\right) \sigma_{k}(z) .
$$

As in the proof of Lemma 4,

$$
\begin{aligned}
\mid C_{n-k}\left(\lambda_{k+1} ;\right. & \left.z_{k}^{(n)}, \ldots, z_{n-1}^{(n)} ; \lambda_{k+1}, \ldots, \lambda_{n}\right) \mid \\
& \leq((W+1) / W)\left(1+r_{1}\right)\left(1+r_{2}\right) \cdots\left(1+r_{n-1}\right) \vdots((W+1) / W) K,
\end{aligned}
$$

for $0 \leq k \leq n$. Therefore for $n \geq N$ and $z \in D, \quad\left|F_{n}(z)\right| \leq((W+1) / W) K \sum_{k=0}^{n}\left|\sigma_{k}(z)\right|$. Noting that $\left\{\sigma_{k}\right\}$ is a basis, the sequence $\left\{F_{n}\right\}_{n=N}^{\infty}$ is uniformly bounded on compact subsets of $D$. Therefore, there exists a set $S$ of positive integers such that the sequence $\left\{F_{n}\right\}_{n} \in S$ converges uniformly on compact subsets of $D$ to a function $F \in \mathbb{G}_{1}$. For each nonnegative integer $k$, the sequence $\left\{\Delta^{k} F_{n}\right\}_{n} \in S$ converges uniformly on compact subsets to $\Delta^{k} F$, by continuity of the map $f \rightarrow$ $\Delta^{k} f$. Since $\Delta^{k} F_{n}\left(z_{k}^{(n)}\right)=0, n \in S$, uniform convergence implies that $\Delta^{k} F$ has a zero on the circle $|z|=W$.

To complete the construction, there remains to show $F \not \equiv 0$. Since $F(z)=$ $F\left(\lambda_{1}\right)+\sum_{k=1}^{\infty} \Delta^{k} F\left(\lambda_{k+1}\right) \sigma_{k}(z)$, it suffices to show that $\Delta^{N} F\left(\lambda_{N+1}\right) \neq 0$. By (3.5).

$$
\Delta^{N} F\left(\lambda_{N+1}\right)=\lim _{n \in S} C_{n-N}\left(\lambda_{N+1} ; z_{N}^{(n)}, \ldots, z_{n-1}^{(n)} ; \lambda_{N+1}, \ldots, \lambda_{n}\right) .
$$

For each $n \geq N+2,(3.4),(1.7)$ and (2.12) imply that

$$
\begin{aligned}
\left|C_{n-N}\left(\lambda_{N+1} ; z_{N}^{(n)}, \cdots, z_{n-1}^{(n)} ; \lambda_{N+1}, \cdots, \lambda_{n}\right)\right| \\
\quad \geq\left|\frac{z_{N}^{(n)}-\lambda_{N+1}}{z_{N}^{(n)}}\right|\left\{\beta-\sum_{\substack{N+2 \leq p_{1}<\cdots<p_{m} \leq n-1 \\
1 \leq m \leq n-N-2}} r_{p_{1}}{ }^{r} p_{2} \cdots r_{p_{m}}\right\} .
\end{aligned}
$$

Writing $\tau_{n}=r_{N+2}+r_{N+3}+\cdots+r_{n-1}$, the previous inequality implies

$$
\begin{aligned}
\left|C_{n-N}\left(\lambda_{N+1} ; z_{N}^{(n)}, \cdots, z_{n-1}^{(n)} ; \lambda_{N+1}, \cdots, \lambda_{n}\right)\right| \\
\geq \frac{W-W \cdot 2}{W}\left\{\beta-\tau_{n}-\tau_{n}^{2}-\cdots-\tau_{n}^{n-N-2\}}\right. \\
\geq \frac{1}{2}\left\{\beta-\sum_{j=1}^{\infty}\left(\sum_{n=N}^{\infty} r_{n}\right)^{j}\right\} \geq \frac{1}{2}\left\{\beta-\sum_{j=1}^{\infty}\left(\frac{\beta}{2+\beta}\right)^{j}\right\}=\frac{\beta}{4}
\end{aligned}
$$

for each $n \in S$ with $n \geq N+2$, and it follow that $\left|\Delta^{N} F\left(\lambda_{N+1}\right)\right| \geq \beta / 4>0$.

Remark. Fix $\rho>0$ and define $C_{\lambda, \rho}$ to be the supremum of numbers $c>0$ 
such that if $f \in \mathbb{G}_{\rho}$ and each of $\Delta_{\lambda}^{k} f(z), 0 \leq k<\infty$, has a zero in $|z| \leq c$, then $f \equiv 0$. By taking $0<R<\rho$ in Theorem 4, we see that $C_{\lambda, \rho} \geq \rho W$ for all $\lambda \in c_{0}$. If $\lambda \in l_{1}$, let $\mu=\rho^{-1} \lambda=\left(\rho^{-1} \lambda_{1}, \rho^{-1} \lambda_{2}, \ldots\right)$ and construct the extremal function $F \in \mathbb{G}_{1}$ with respect to the sequence $\mu$. Define $G(z)=F(z / \rho)$. Then $G \in \mathbb{Q}_{\rho}$, $G \not 0$, and each of $\Delta_{\lambda}^{k} G(z)$ has a zero on $|z|=\rho W$. It follows that $C_{\lambda, \rho}=\rho W$ for $\lambda \in l_{1}$.

Added in proof. The authors wish to thank Professor J. L. Franks for pointing out this problem.

\section{REFERENCES}

1. J. D. Buckholtz, Zeros of partial sums of power series. II, Michigan Math. J. 17 (1970), 5-14. MR $41 \# 3718$.

2. J. D. Buckholtz and J. L. Frank, Whittaker constants, Proc. London Math. Soc. 23 (1971), 348-370.

3. J. D. Buckholtz and J. K. Shaw, Zeros of partial sums and remainders of power series, Trans. Amer. Math. Soc. 166 (1972), 269-284.

4. P. J. Davis, Interpolation and approximation, Blaisdell, New York, 1963. MR 28 \#393.

5. M. M. Dragilev, On the convergence of the Abel-Gončarov interpolation series, Uspehi Mat. Nauk 15 (1960), no. 3 (93), 151-155. (Russian) MR 22 \#4839.

6. J. L. Frank and J. K. Shaw, Abel-Gončarov polynomial expansions, J. Approximation Theory (to appear).

7. E. Hille, Analytic function theory. Vol. 1, Introduction to Higher Math., Ginn, Boston, Mass., 1959. MR 21 \#6415.

8. G. Köthe, Topological vector spaces. I, Die Grundlehren der math. Wissenschaften, Band 159, Springer-Verlag, New York, 1959. MR 40 \#1750.

9. A. L. Perissini, Concerning the order structure of Köthe sequence spaces. II, Michigan Math. J. 11 (1964), 357-364.

10. M. Pommiez, Sur les différences divisées successives et les restes des séries de Newton généralisées, Ann. Fac. Sci. Univ. Toulouse (4) 28 (1964), 101-110. MR 33 \#7559b.

11. H. H. Schaefer, Topological vector spaces, Macmillan, New York, 1966. MR 33 \#1689.

DEPARTMENT OF MATHEMATICS, VIRGINIA POLYTECHNIC INSTITUTE AND STATE UNIVERSITY, BLACKSBURG, VIRGINIA 24061 